Draft VERSiOn MAY 25, 2022

Preprint typeset using $\mathrm{L}^{\mathrm{A}} \mathrm{T} \mathrm{E}$ X style emulateapj v. 11/10/09

\title{
FORMATION OF PLANETARY CORES AT TYPE I MIGRATION TRAPS
}

\author{
Zsolt SÁNdor $^{1}$, Wladimir Lyra ${ }^{2}$, And CORnelis P. Dullemond ${ }^{1,3}$ \\ Draft version May 25, 2022
}

\begin{abstract}
One of the longstanding unsolved problems of planet formation is how solid bodies of a few decimeters in size can "stick" to form large planetesimals. This is known as the "meter size barrier". In recent years it has become increasingly clear that some form of "particle trapping" must have played a role in overcoming the meter size barrier. Particles can be trapped in long-lived local pressure maxima, such as those in anticyclonic vortices, zonal flows or those believed to occur near ice lines or at dead zone boundaries. Such pressure traps are the ideal sites for the formation of planetesimals and small planetary embryos. Moreover, they likely produce large quantities of such bodies in a small region, making it likely that subsequent N-body evolution may lead to even larger planetary embryos. The goal of this Letter is to show that this indeed happens, and to study how efficient it is. In particular, we wish to find out if rocky/icy bodies as large as $10 M_{\oplus}$ can form within 1 Myr, since such bodies are the precursors of gas giant planets in the core accretion scenario.

Subject headings: planets and satellites: formation — protoplanetary disks — planet-disk interactions - methods: numerical
\end{abstract}

\section{INTRODUCTION}

There are a number of major unsolved problems in the theory of rocky planet formation. One is the infamous "meter size barrier" problem (Blum \& Wurm 2008): When dust aggregates reach sizes beyond roughly $1 \mathrm{~cm}$, they partially decouple dynamically from the gas and can reach relative velocities with respect to other particles of up to $50 \mathrm{~m} / \mathrm{s}$ (Weidenschilling \& Cuzzi 1993). Any such collision will likely lead to fragmentation and/or erosion rather than growth. In addition, these bodies tend to drift radially inward toward the star at a high speed, and thus quickly get lost to the planet formation process (Brauer et al. 2008). Moreover, as laboratory experiments and numerical modeling (Zsom et al. 2010) show, already at millimeter sizes the dust aggregates stick insufficiently well for coagulation to continue. Despite many theoretical studies aimed at solving this problem, this issue is still wide open. In recent years a new line of thought has emerged which invokes various local particle trapping mechanisms to solve it. Cuzzi et al. (2008) propose that particle concentrations in a turbulent disk occurring naturally at small eddy scales can, statistically, sometimes lead to self-gravitating "sandpiles" that gradually condense to form planetesimals of 1 to $100 \mathrm{~km}$ radius. The particles must have grown to millimeter sizes before this process sets in, but the meter size barrier is thus easily overcome. Johansen et al. (2007) showed that particle trapping and subsequent gravitational contraction of particle clouds can also happen at the scale of the largest turbulent eddies, leading to bodies between $100 \mathrm{~km}$ and $1000 \mathrm{~km}$ in size. This mechanism can in fact also act on

${ }^{3}$ Institut für Theoretische Astrophysik, Universität Heidelberg, Heidelberg, Germany
}

scales much larger than the turbulent eddy scale. For instance, Barge \& Sommeria (1995) and Klahr \& Henning (1997) showed that particles tend to get trapped in anticyclonic vortices, if they exist in protoplanetary disks. Varnière \& Tagger (2006) and Terquem (2008) showed with alpha disk models (Shakura \& Sunyaev 1973) that pressure enhancements are expected at dead zone boundaries because of the difference in turbulent viscosity. Such enhancements were confirmed in the MHD simulations of Kato et al. (2010). Kretke \& Lin (2007) suggested that similar pressure ridges form at the snow line in the disk (see discussion by Dzyurkevich et al. 2010), while Johansen et al. (2009) showed that an "inverse cascade" of magnetorotationally driven turbulence will lead to large scale pressure bumps in disks.

Another extensively studied problem is the retention of protoplanets in the disk for a sufficiently long time for the core accretion process. This requires at least 10 times slower type I migration speed than estimated from analytical theory (Alibert et al. 2005). In recent years, however, the understanding of type I migration has changed considerably (e.g. Paardekooper \& Mellema 2006; Klev et al. 2009; Paardekooper et al. 2010). As Morbidelli et al. (2008) have demonstrated by using a proper surface density profile in their hydro simulations a planet trap appears, which helps forming massive rocky/icy bodies and prevents their migration to the central star. We will hereafter adopt the (non-standard) nomenclature "terrestrial planet" and "planetary core" for rocky/icy bodies of mass below and above $10 M_{\oplus}$, respectively.

Many of the above ideas were combined in a single model by Lyra et al. (2008, 2009). The model explores what happens at dead zone boundaries, where the density enhancement was predicted by Varnière \& Tagger (2006) and Inaba \& Barge (2006) to be unstable to the Rossby Wave Instability (Lovelace et al. 1999), which in turn leads to the formation of large scale anticyclonic vortices. Particles of about $\mathrm{cm}$ to meter in size subsequently drift into the vortices and form gravitationally 
bound clumps of solids, ranging between the masses of the Moon and Mars.

The present paper follows up on that work. Lyra et al. (2008, 2009) explored only the formation mechanism, since the hydrodynamical models are too burdensome to follow the evolution for longer than a few thousand of years (which is but a small fraction of the lifetime of the gas-rich phase of the disk). A large number of massive embryos are formed that all initially have semimajor axes very close to the radial location of the pressure bumps where they were formed. The location near these "planet factories" are thus quickly overpopulated with embryos. Mutual collisions and merging events are naturally expected in such scenario. It would be of a great interest to determine how the ensemble develops over a long time scale.

In this paper we follow the N-body evolution of the heaviest embryos of the swarm that were produced during the first few hundred orbits in the above model. We include the gravitational interaction between the embryos and the gas in the disk which leads to type I migration. We account for it by applying the analytic formulae used by Lyra et al. (2010), and developed originally by Paardekooper et al. (2010). The model is detailed in the next section.

\section{IMPLEMENTATION OF TYPE I MIGRATION}

To integrate numerically the differential equations of the gravitational N-body problem we developed a Bulirsch-Stoer integrator, which can handle collisions between nearby bodies. When the mutual distance between the center of mass of two nearby bodies is less than the sum of their physical radii, the bodies collide and merge. The physical radius of a body is calculated using its mass assuming a $2 \mathrm{~g} / \mathrm{cm}^{3}$ bulk density for the whole embryo population. The mass and the initial velocity of the newly formed body are calculated assuming perfectly inelastic collision using the center of mass approximation.

In what follows, we describe briefly how the dissipative forces for type I migration have been implemented in our N-body code knowing the surface density and temperature profiles of the disk.

In isothermal disks the total torque $\Gamma$ experienced by a body can be written as

$$
\Gamma / \Gamma_{0}=-0.85-\alpha-0.9 \beta,
$$

where $\alpha$ and $\beta$ are the negative of the local surface density $(\Sigma(r))$ and temperature $(T(r))$ gradients

$$
\alpha=-\frac{d \log \Sigma}{d \log r}=-\frac{r}{\Sigma} \frac{d \Sigma}{d r}, \beta=-\frac{d \log T}{d \log r}=-\frac{r}{T} \frac{d T}{d r},
$$

and $\Gamma_{0}=(q / h)^{2} \Sigma(r) r^{4} \Omega(r)^{2}$ (see Paardekooper et al. 2010). Here $q=m / M_{*}$ is the body to star mass ratio, $h=H(r) / r$ is the disk constant aspect ratio $(H(r)$ being the disk's vertical thickness), and $\Omega$ the Keplerian angular velocity.

Using Equations (1) and (2), the total torque $\Gamma$ can be easily determined, which enables the calculation of the body's radial migration speed as follows:

$$
\dot{r}=\dot{L}\left(\frac{d L}{d r}\right)^{-1}=2 r \frac{\Gamma}{L},
$$

where $L=m \sqrt{G M_{*} r}$ is the angular momentum of the

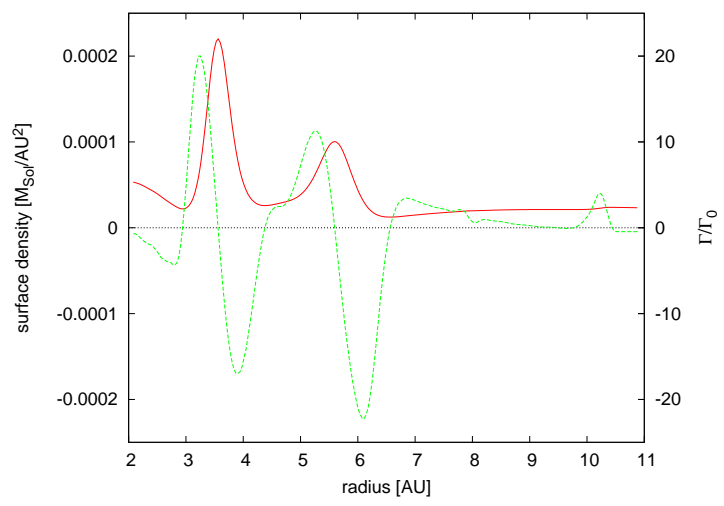

FIG. 1.- The surface density profile plotted with solid red (dark) line, the dimensionless torque $\Gamma / \Gamma_{0}$ with the dotted green (light) line. The zero torque places are practically at the two "peaks", and the three "valleys". If $\Gamma / \Gamma_{0}>0$ outward, if $\Gamma / \Gamma_{0}<0$ inward migration occur.

body. The migration timescale is $\tau_{\text {migr }}=-r / \dot{r}$, which using Equation (3) reads

$$
\tau_{\text {migr }}=-\frac{r}{\dot{r}}=\frac{1}{m} \frac{h^{2} M_{*}^{3 / 2}}{(1.7+2 \alpha+1.8 \beta) \Sigma \sqrt{r G}} .
$$

Note that a negative $\tau_{\text {migr }}$ means outward migration. In addition to the inward migration, a body also feels the strong damping effects of the disk on its orbital eccentricity and, since the N-body part of our model is fully $3-\mathrm{D}$, its orbital inclination. The corresponding damping timescales are $\tau_{\text {ecc }}, \tau_{\text {inc }} \sim h^{4} \tau_{\text {migr }}$ (see the exact formulae in Tanaka \& Ward (2004)).

Knowing the timescales $\tau_{\text {migr }}, \tau_{\text {ecc }}$, and $\tau_{\text {inc }}$, the corresponding forces can be implemented in the N-body code. In our code we applied the formula of Cresswell \& Nelson (2008), which for the $i$ th body is

$$
\ddot{\mathbf{r}}_{i}=\ddot{\mathbf{r}}_{i, \text { grav }}-\frac{\dot{\mathbf{r}}_{i}}{2 \tau_{\text {migr }}}-2 \frac{\left(\dot{\mathbf{r}}_{i} \cdot \mathbf{r}_{i}\right) \mathbf{r}_{i}}{r_{i}^{2} \tau_{\text {ecc }}}-\frac{\left(\dot{\mathbf{r}}_{i} \cdot \mathbf{k}\right) \mathbf{k}}{\tau_{\text {inc }}},
$$

where $\mathbf{k}$ is the unit vector in the $z$-direction and $\ddot{\mathbf{r}}_{i \text {,grav }}$ is the gravitational acceleration induced by all the other bodies (the N-body force).

\section{PHYSICAL BACKGROUND OF OUR SIMULATIONS}

As initial conditions of our N-body experiments we used the embryo population formed during the hydrodynamical simulations of Lyra et al. (2008, 2009). In that hydro simulation the initial surface density and temperature profiles followed power law distributions $\Sigma \sim r^{\alpha}$ with $\alpha=-0.5$, and $T \sim r^{\beta}$ with $\beta=-1$. The density profile changed considerably during the simulations, but due to the local isothermal disk assumption, $\beta=-1$ was kept fixed. For the torque calculations we used the azimuthally averaged surface density profile obtained at the end of the hydro simulations, see Fig. 1. To keep the problem tractable we fix the gas density profile in time as it appears in the figure. The dimensionless torque $\Gamma / \Gamma_{0}$ is also displayed. Notice that it reaches values as high as \pm 10 . We caution that Equation (1) was derived for smooth disks, and may thus not be unproblematic when used for strongly irregular disks like the one in our model. But at present this is the only tractable way to treat the problem of type I migration of many bodies over a long timescale. 
In Fig. 1 the two density peaks appear at the inner and the outer edge of the dead zone, and correspond to the large vortices. Since $p=\Sigma c_{s}^{2}$ ( $p$ is the verticaly integrated pressure and $c_{s}$ is the local sound speed), and the temperature distribution $(T(r))$ is fixed, the density peaks correspond approximately to pressure maxima.

The embryos form in narrow regions around the two pressure peaks (corresponding to the density peaks of Fig. 1), and they are expected to disperse somewhat due to mutual gravitational scattering effects. The peaks are very nearby to zero-torque radii. They act as planet traps ("planetary convergence zones' '4) because inward migration occurs for radii larger than the peak's location, whereas outward migration occurs for smaller radii than that. There are zero torque places in the density valleys as well. But, in contrary to the peaks, they have repulsive character.

On the other hand, the embryos form on a relatively short timescale, during 200 periods (measured at Jupiter's orbital distance) consuming practically the solid content of the (strongly truncated) disk used in the hydro simulations. If we consider a realistic 100-200 AU disk, repeated formation of the planetary embryos can be expected as long as the large vortices at the dead zone edges exist, and the disk contains enough solid material with size between $1 \mathrm{~cm}-1 \mathrm{~m}$. These particles are very strongly affected by the gas drag, and therefore are drifted inward quickly and continuously to the embryo forming vortices as long as gas exists in the system. In this way the outer part of the disk acts as a large reservoir of solid material and due to the strong radial drift the large vortices, which are working as "planet factories" are replenished continuously with "raw" material. The continuous embryo formation in the "planet factory" is an important part of our physical assumptions. In our N-body runs we do not intend to model this process, for feasibility reasons. But to mimic the continuous production of new bodies, we simply inject Mars-mass embryos stochastically into the pressure trap region, given a production rate which we take as a parameter of our model. The new bodies are assumed to form only at the outermost pressure trap (the one at the outer edge of the dead zone). We do this for two reasons: One is to make the proces more "clean" and understandable, focusing on a single pressure trap only. The other is that it is reasonable to assume that the influx of dust from the very outer disk regions gets captured by the outer pressure trap, thus choking the inner pressure trap off from the supply of new material.

Armed with the above ideas, we performed two long term N-body simulations to see how terrestrial planets, and even planetary cores can be formed around the zero torque places of the disk.

\section{RESULTS AND DISCUSSION}

Since we are interested in the formation of massive planets, we considered as initial embryo population of our N-body runs only the 10 most massive bodies, formed during one of the hydrodynamical simulations of Lyra et al. (2009) in the outermost pressure trap. We performed two simulations: In the first one (simulation

\footnotetext{
4 The nomenclature convergence zone was suggested by C. Mordasini, and adopted here.
}

A) a $1 / 3 M_{\oplus}$ body 5 was assumed to form at irregular time intervals according to a Poisson distribution with a rate of $2 \times 10^{-4}$ year $^{-1}$. In the second one (simulation $\mathrm{B}$ ), a $1 / 6 M_{\oplus}$ body was assumed to form at time intervals also following Poisson distribution with a rate $4 \times 10^{-4}$ year $^{-1}$. The results of simulations A and B are shown respectively in Figs. 2 and 3 .

At the beginning of our N-body simulations close approaches, scattering events and collisions happened between the initial embryos, after which a multiple mean motion resonant structure formed. Subsequently, when new bodies got inserted into the pressure traps, this resonant structure is perturbed and new scattering events and mergers happen, leading to ever larger masses. The whole structure of the resonantly interacting planets is broadening, because of the ever increasing masses of the planets and the increasing number of them. At around $10^{5}$ to $1.7 \times 10^{5}$ years the planets which have been resonantly pushed the farthest away from the pressure bump reach the edges of the type I migration convergence zone $(4.4 \lesssim r \lesssim 6.6 \mathrm{AU})$ and start to migrate away from their birthplace. For the ones that migrate inward, they get again trapped, this time in the innermost migration convergence zone.

Through collisions some of the bodies are able to increase their masses by accreting either the newly formed, or the already existing embryos. After $4 \times 10^{5}$ years-long numerical integration, a planetary core of $10 M_{\oplus}$ was formed both in simulation A and B. Thus, regarding the final mass of the planetary core formed, there is no significant difference between the results of simulations A and B. Comparing the evolution of the semi-major axes in simulations A and B, it can be clearly seen that in case A the evolution of the system is "smoother" than in case B. The reason for this is that in simulation $\mathrm{A}$ a smaller number of bodies is involved than in simulation B. However, the final mass of the planetary core formed in both simulations is almost the same after $\sim 4 \times 10^{5}$ years. This means that the most important parameter of our simulations is the amount of mass injected during a given time.

We call attention to the fact that, in some aspects, the hydro simulations of Morbidelli et al. (2008) are similar to ours. We therefore briefly compare the methods and results of the two works. In that study the $2 \mathrm{D}$ hydro code FARGO (Masset 2000) has been used, and the N-body integrator was implemented in 3D, in which the planet's inclination has been damped according to Tanaka \& Ward (2004). During the hydro simulations the steep surface density profile halted the migration of 10 embryos (having each a mass of $1 M_{\oplus}$ ), but the resonant structure between them survived only temporarily, and some of the embryos were able to collide and merge without any additional perturbative event. In our case, if there would have been no continuous formation of embryos, the resonant structure would be stable, preventing the bodies from further collisions. There are however a few major differences between the physical models. The static surface density profile used in our study (providing a strong constant torque), differs from that in the hydro simulations of Morbidelli et al. (2008). They use an

5 Corresponding to the mass of the largest body obtained by Lyra et al. (2009). 

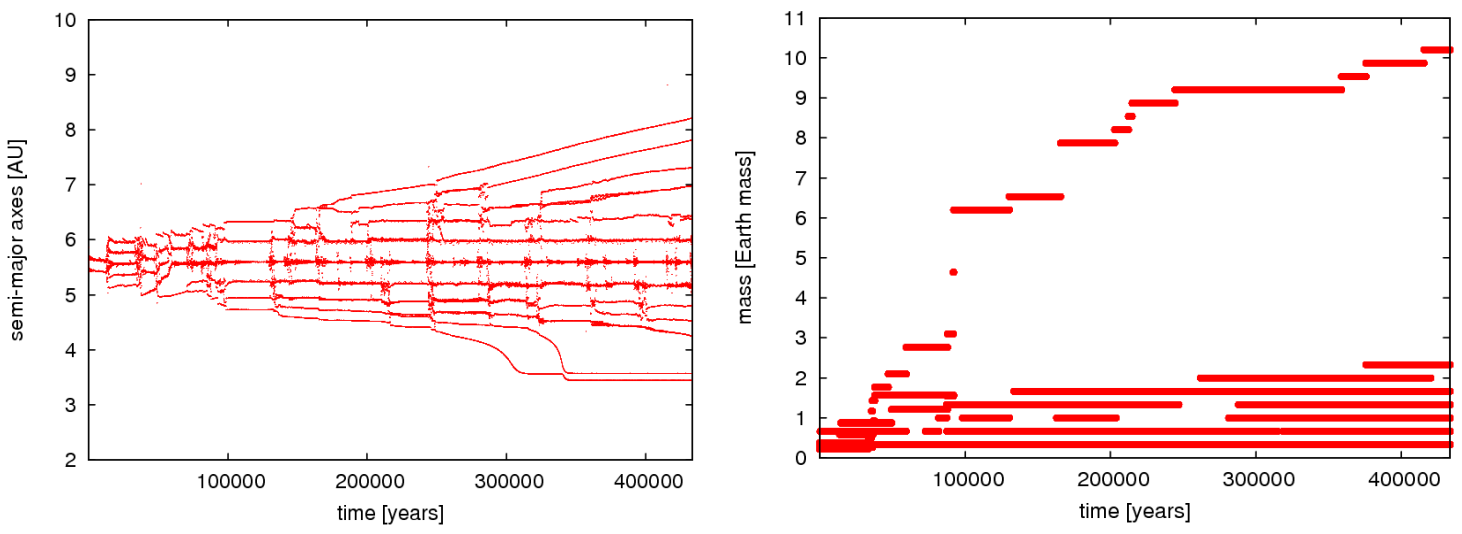

Fig. 2.- Left: Evolution of the semi-major axes during the simulation A. Right: The corresponding overall mass accretion of the embryo population. The most massive body is a planetary core with mass $\approx 10 M_{\oplus}$.
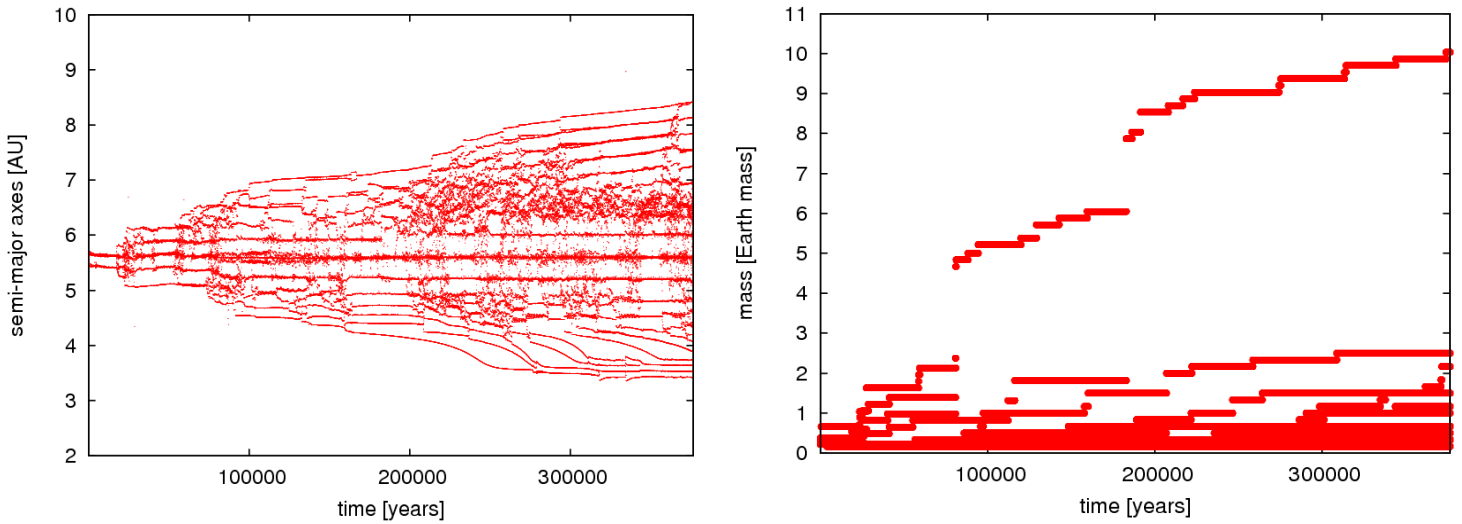

Fig. 3.- The same as in Fig. 2 for simulation B.

evolving 2D disk, in which the back reaction of the bodies to the disk influences the local gas density distribution, thus the torques are probably non-constant. The torques are also weaker, since the surface density distribution is apparently much smoother than in our case. If instead of a strong static torque, we would use a weaker and oscillating one, the protecting resonant structure might be destroyed, enabling even a more rapid formation of a planetary cor 6. Compared to Morbidelli et al. (2008) the novelty of our approach is that we consider the continuous formation of embryos assembled at the pressure trap, and we study their long-term evolution. We also use smaller building blocks, and follow the core formation process through a broader mass range of bodies involved in it.

In the core accretion scenario, the formation of gas giant planets can only occur if planetary cores are grown still in the gas rich phase. As our simulations show, the time required for the assembly of a $10 \quad M_{\oplus}$ core is $\lesssim 0.5 \times 10^{6}$ years in the framework of our settings. Since the lifetime of the gas disk is expected to be $\approx 5$ Myr, there is significant time left for accretion of nebular gas, completing the formation of a gas giant planet (Alibert et al. 2005).

\footnotetext{
${ }^{6}$ The torque oscillation would be due to the fact that the trapping zone in fact consists of a couple of large vortices arranged in a circle (see Lyra et al. 2008), which means that, depending on the azimuthal location of the planet compared to the locations of those vortices, the planet may experience different torques.
}

\section{SUMMARY AND OPEN QUESTIONS}

In this Letter we investigated the possibility to form large bodies in protoplanetary disks at such places where the torques responsible for type I migration vanish. We assumed that at the edges of the dead zone of the disk large vortices develop that can collect the (from centimeter to meter sized) solid content of the disk. Through the self gravity of these overdense regions of solids, relatively large embryos are formed, with masses up to $1 / 3 M_{\oplus}$ (Lvra et al. 2008, 2009).

Due to the particular surface density distribution obtained from the above mentioned hydro simulations the embryos stay trapped close to their birthplaces because the location of the type I migration convergence point is very close to the location of the pressure trap. The embryos capture each other into mean motion resonances, entering into a very robust protective configuration against further collisions. This resonant structure is, however, perturbed from time to time when a new massive embryo forms in one of the giant vortices. During these perturbative events, the embryos can collide and, by merging events, form planetary cores as massive as $10 M_{\oplus}$. The whole process takes $\lesssim 0.5 \mathrm{Myr}$.

We stress that the proposed scenario should also work in disk models where the pressure trap emerges by other mechanics, such as at the iceline, as in Kretke \& Lin (2007); or in zonal flows, as in Johansen et al. (2009).

Besides cores of giant planets, other massive terrestrial 
planets can also be formed, which by scattering at later epochs may lead to the formation of a complete planetary system. The escape of the bodies from the zero torque places can also be caused by the slowly forming giant planet, which by opening a gap will change the surface density profile. On the flip side, however, this scenario would predict that, if the trap is located beyond the ice line, all rocky planets of the resulting planetary system should be ice- or ocean planets, which for our solar system is clearly not the case. Further research is thus required to investigate these issues.

It is clear, however, that our present model is still very simplified, and further study is required to verify our proposed scenario for the formation of rocky/icy planetary cores. For instance Morbidelli et al. (2008) have shown that, due to the large vortices at the planet trap, the semi-major axes of the embryos oscillate. This means that the position of the zero torque point of any given planet is not constant in time. Thus the dynamics of the whole embryo population is perturbed, which may result in more effective collisions of bodies at the convergence zone, shortening the time to form a planetary core. On the other hand, the perturbations induced in the disk by these planets may shake up and destroy the tranquil nature of the pressure bumps in which embryos are supposed to be formed, thus perhaps quenching the formation of new embryos. Also, the planets that are pushed away from the convergence zone to larger radial distances may form a barrier to dust drifting inward from the outer parts of the disk. All of these questions require further study, and much more detailed modeling. But considering that the combined dust- and planet-migration trap scenario we propose here has the potential to solve both the meter-size barrier problem and the time scale problem of oligarchic growth, it seems worthwhile to invest substantial effort in studying planet formation along these lines.

We thank the anonymous referee for useful suggestions.

\section{REFERENCES}

Alibert, Y., Mordasini, C., Benz, W. \& Winisdoerffer, C. 2005 , A\&A, 434, 343

Barge, P. \& Sommeria, J. A\&A, 295, L1-L4

Blum, J. \& Wurm, G. 2008, ARA\&A, 46, 21

Brauer, F., Dullemond, C. P. \& Henning, Th. 2008, A\&A, 480, 859

Cresswell, P. \& Nelson, R. P. 2008, A\&A, 482, 677

Cuzzi, J. N., Hogan, R. C. \& Shariff, K. 2008, ApJ, 687, 1432

Dzyurkevich, N., Flock, M., Turner, N. J., Klahr, H. \& Henning, Th. 2010, A\&A, 515, 70

Inaba, S. \& Barge, P. 2006, ApJ, 649, 415

Johansen, A., Oishi, J. S., Mac Low, M.-M., Hubert, K., Henning, Th. \& Youdin, A. 2007, Nature, 448, 1022

Johansen, A., Youdin, A. \& Klahr, H. 2009, ApJ, 697, 1269

Kato, M. T., Fujimoto, M. \& Ida, S. ApJ, 714, 1155

Klahr, H. \& Henning Th. 1997, Icarus, 128, 213

Kley, W., Bitsch, B. \& Klahr, H. (2009) A\&A, 506, 971

Kretke, K. A., Lin, D. N. C. 2007, ApJ, 664, L55-L58

Lovelace, R. V. E., Li, H., Colgate, S. A., \& Nelson, A. F. 1999, ApJ, 513, 805L
Lyra, W., Johansen, A., Klahr, H. \& Piskunov, N. 2008, A\&A, 491, L41-L44

Lyra, W., Johansen, A., Zsom, A., Klahr, H. \& Piskunov, N. 2009, A\&A, 497, 869

Lyra, W., Paardekooper, S.-J. \& Mac Low, M.-M. 2010, ApJ, 715, L68-L73

Masset, F. 2000, A\&AS, 141, 165

Matsumura, S., Pudritz, E. R. \& Thommes, E. W. 2009, ApJ, 691,1764

Morbidelli, A., Crida, A., Masset, F. \& Nelson, R. 2008, A\&A, 478,929

Paardekooper, S.-J. \& Mellema, G. 2006, A\&A, 459, 17

Paardekooper, S.-J., Baruteau, C., Crida, A. \& Kley, W. 2010, MNRAS, 401, 1950

Shakura, N. I. \& Sunyaev, R. A. 1973, A\&A, 24, 337

Tanaka, H. \& Ward, W. R. 2004, ApJ, 602, 388

Terquem, C. E. J. M. L. J. 2008, ApJ, 689, 532

Varnière, P. \& Tagger, M. 2006, A\&A, 446, 13

Weidenschilling, S. J. \& Cuzzi, J. N. 1993, Protostars and planets III, pp. 1031-1060. Eds: E. H. Levy \& J. I. Lunine

Zsom, A., Ormel, C. W., Gütler, C., Blum, J. \& Dullemond, C. P. 2010, A\&A, 513, 57 